\title{
Understanding immunity requires more than immunology
}

\author{
Kevin J Tracey \\ Center for Biomedical Science, Feinstein Institute for Medical Research, Manhasset, New York, \\ USA
}

Kevin J Tracey: kjtracey@nshs.edu

\begin{abstract}
Acetylcholine and related neurotransmitters appeared with unicellular life forms, millions of years before innate immunity. Tools and insights are now available for understanding how the evolving nervous system influenced the development of immunity.
\end{abstract}

Ten years ago, on page 1 of the first volume of Nature Immunology, the editors noted that "immunology has always been driven by fundamentally interesting questions." Today, the intrigue continues. How is immunity acquired, controlled and maintained? How did it evolve? What is the physiological role of immunity during the host response to infection, cancer, aging and injured tissue repair? What can the molecular mechanisms of immunity teach about metabolism, the activity of complex systems and new approaches for developing targeted therapeutics?

There can be no quarrel with the argument that the field addresses interesting questions, but I propose that it is also driven by society's need for new therapies. From its origins, progress in immunology can be tracked by the development of new therapeutics and clinical successes that elevated the importance of the field. Often these advances have been inextricably linked to new knowledge, technologies and concepts provided by other fields. The validation of the germ theory of disease in the nineteenth century, coupled with parallel advances in microbiology, enabled the development of specific vaccines. Similarly, knowledge of immunity in the twentieth century, coupled with advances in biochemistry, enabled the development of blood transfusions and organ transplantation. Most recently, the elucidation of the cytokine theory of disease, coupled with advances in monoclonal antibody technology and molecular biology, enabled development of cytokine therapeutics that revolutionized therapy for millions of patients with rheumatoid arthritis, psoriasis, cancer, inflammatory bowel disease or other clinical syndromes ${ }^{1}$. Now, as experience with these disease-modifying agents grows, new mechanisms for other potentially treatable diseases are being explored. The established precedent is that the development of therapeutics that target the immune system has been accelerated by advances in allied scientific fields, practical gains that have diminished disease morbidity and mortality and identified more interesting new questions.

Competing Financial Interests: The author declares competing financial interests: details accompany the full-text HTML version of the paper at http://www.nature.com/natureimmunology/. 
This commentary addresses what may come next. What are the unresolved problems that might be solved by using tools from other fields heralding future advances in immunology? Like most scientists, loath to forecast the future, I greatly prefer focusing on the data at hand and learning from what it can teach. But for the purpose of this commentary, I will consider some immunology topics that are now intersecting with advances in allied fields and imagine frameworks for accelerated progress based on the need to treat common diseases.

\section{Reflexes control innate immunity}

Once it was established that cytokines are able to mediate sickness syndromes and lethal damage, interest turned toward answering questions about normal feedback mechanisms that restrain cytokine production and toxicity to maintain health. Anti-inflammatory mediators were identified, including hormones in the hypothalamic-pituitary-adrenal axis, as well as soluble cytokine receptors and other cytokine suppressing factors. The accepted theory was that inflammation is regulated by an inhibitory humoral network that prevents excessive inflammation and encourages its resolution. The relatively straightforward concept of antiinflammatory cascades fit nicely with the generally accepted view that the immune system is a distributed network of independent cells modulated by humoral and paracrine factors and by direct cell-to-cell interactions. It came as more of a surprise when advances in neurophysiology identified neural circuits that directly regulate innate immunity. The concept that action potentials, traveling in neurons and coordinated by the central nervous system, control the activity of the immune system was somewhat antithetical to the belief that the immune system is autonomous ${ }^{2}$.

It may be illustrative to consider, by analogy, that the activity of other complex organ systems is regulated by neural reflexes (for example, heart rate and blood pressure in the cardiovascular system). These reflexes are composed of three components: a sensory neural path, which relays information to the central nervous system; a centrally located interneuronal relay center; and an efferent path that delivers corrective or compensatory neural signals that regulate the activity of the innervated system. The inflammatory reflex, a prototypical neural circuit that modulates innate immunity, is activated by the presence of cytokines or other inflammatory products in tissues that triggers afferent action potentials traveling in the vagus nerve. The ascending information is relayed to brainstem nuclei that control efferent neural signals in the form of action potentials transmitted back to the periphery via the vagus nerve. This information is relayed to the spleen and other cytokineproducing organs, where cytokine expression is inhibited by a molecular mechanism that requires signal transduction via the nicotinic a7-acetylcholine receptor subunit. Animals deficient in either vagus nerve activity or the nicotinic a 7 -acetylcholine receptor subunit are exquisitely sensitive to inflammatory challenges because they overproduce cytokines. The cytokine-braking mechanisms exerted by the motor arc of the inflammatory reflex, called the 'cholinergic anti-inflammatory pathway', prevents the damaging consequences of excessive innate immunity. This neurophysiological approach taught that results obtained with isolated cells of the immune system may not be used to accurately predict their activity in an intact host because the cellular biology, physiology and metabolism of a denervated system can not recapitulate the responses of an innervated, reflexively controlled system. Thus, in the example cited here, stimulated monocytes and macrophages produce 
substantially more cytokines if they are deprived of the regulating influence of the cholinergic anti-inflammatory pathway, so knowledge of the activity of this neural circuit is needed to understand innate immunity in the host.

Advances in the fields of biomedical and electrical engineering enabled development of new neurostimulators that can be implanted selectively in the brain or along the course of the vagus nerve or other nerves to modulate the activity of neural circuits. Preclinical studies established that vagus nerve activity is lower in the presence of inflammatory diseases, a result that is consistent with enhanced cytokine release and tissue injury that underlies pathogenesis. Neuromodulating devices implanted in thousands of patients to treat seizure disorder and depression have an excellent safety profile $\mathrm{e}^{3}$. The time has now arrived to address the question of whether neuromodulating devices can be used to treat inflammatory diseases mediated by cytokines. Preclinical studies indicate that controlled electrical modulation of vagus nerve activity inhibits cytokine release and confers considerable protection from tissue injury in widely used models of arthritis, sepsis, inflammatory bowel disease, ischemia-reperfusion injury and ileus ${ }^{1,2}$. Clinical trials are well within reach and should show whether selective device-based methods to control a regional neural circuit represent a therapeutic option for diseases that until now have been treated by molecules that are delivered and act systemically. As this therapeutic modality is explored, it will be critically important to understand how neural activity modulates other aspects of the immune system, the adaptive immune response and immunological memory. Beyond this, it will be an incremental step from ongoing work to identify other reflexes and neural pathways as targets of neuromodulating devices to control innate and adaptive immunity. And there will probably be new possibilities for exploiting neurotransmitters and their cognate receptors in the development of new pharmaceuticals to control immunity.

\section{Infection and sterile injury coverage}

The field of cytokine biology, with its roots in studies of fever, weight loss and acute-phase protein responses, has been historically coupled to studies of the behavioral and physiological response to infection and injury. Although progress was occasionally slowed by concerns that biological activities were due to bacterial products contaminating the cytokine preparations, eventually it was accepted that highly purified cytokines, devoid of foreign toxins, can directly mediate fever, anorexia, weight loss, altered sleep patterns and social aversion. This constellation of signs, called 'sickness syndrome', is recapitulated by the administration of cytokines into the bloodstream or directly into the brain ${ }^{4}$. Specific cytokine receptors expressed by neurons in the hypothalamus and other brain regions have been linked to the regulation of body temperature, appetite, arousal and other physiological states. Thus, cytokines produced during innate immunity are able to modulate the activity of neural circuits in the central nervous system, which in turn controls the physiological, metabolic and cellular responses of sickness syndrome (Fig. 1).

A major advance in the field was the elucidation of a mechanism by which cytokines activate 'sickness behavior' in the peripheral tissues without directly entering the brain ${ }^{5}$. Specifically, interleukin 1 (IL-1) and other cytokines can trigger the onset of the fever by activating sensory neurons ${ }^{5}$. When the vagus nerves of rats are cut before IL-1 is 
administered into the abdomen, the febrile response to IL-1 fails to occur. The mechanism is dependent on the binding of IL-1 to chemosensitive glomus cells that reside along the course of autonomic nerves ${ }^{6}$. Receptor-ligand interactions stimulate glomus cells to release dopamine, which activates sensory fibers to transmit information about the presence of inflammation into the central nervous system. This elucidated discovered the existence an early neuronal detection system, like a sensory organ for inflammation, and raised the provocative possibility that the nervous system is able to sense microbial invasion and inflammation using mechanisms not classically attributed to the innate immune system; nerves themselves can accomplish the sensory task. Sensory neurons poised to transmit action potentials work together with the epithelial cells and cells of the innate immune system residing on the front lines of the compartmentalized interfaces with the environment to detect invasion or injury. These ascending trains of neural information can provide realtime status reports about microbial products, foreign antigens and inflammatory mediators directly to the nervous system's command centers.

Separate advances in the field of sepsis, the syndrome of systemic inflammation and organ damage that complicates infection or sterile injury, have provided understanding of the mechanisms by which sterile injury activates innate immunity. High-mobility-group box 1 (HMGB1), a ubiquitous protein first identified as a DNA-binding nuclear protein, was later shown to be a necessary mediator of sepsis produced downstream of the early cytokine cascade $^{7,8}$. HMGB1 is actively secreted by monocytes, macrophages and other cells stimulated by exposure to bacterial products or cytokines. The administration of neutralizing antibodies to HMGB1 confers a substantial survival advantage and protection against tissue injury in preclinical models of sepsis, endotoxemia and other models of microbial invasion ${ }^{9}$. An abundant component of cells, it is released in large quantities after sterile injury to the cell from hypoxia, cytotoxicity or physical damage to cell integrity (Fig. 1). HMGB1 is a necessary and sufficient mediator of the pathophysiological response to hemorrhagic shock and reperfusion injury ${ }^{10}$, an example of 'sickness behavior' that occurs in the absence of infection. The administration of neutralizing HMGB1-specific antibodies prevents the development of secondary damage during ischemia-reperfusion injury, which requires HMGB1 signaling through Toll-like receptor 4 (TLR4) ${ }^{10}$. TLR4 is required for HMGB1mediated activation of macrophages, and the molecular binding of HMGB1 with TLR4 and resultant signaling requires a critical redox-sensitive cysteine at position 106 of HMGB1 (ref. 11). Together, these observations have provided a solution to the long-standing question of why patients with sterile injury arising from trauma or ischemia reperfusion develop sepsis-like clinical syndromes that are in many ways indistinguishable from those of patients with microbial infection. The answer is that HMGB1 can activate innate immunity by triggering signal transduction through a portfolio of pattern-recognition receptors that can also be activated by foreign microbial products. This activation culminates in the release of cytokines that mediate the neurological and metabolic manifestations of sickness syndromes that are qualitatively similar regardless of whether cytokine release was elicited by endogenous or exogenous stimulating agents.

This field of endogenous molecules stimulating innate immunity has recently intersected with advances in understanding of the molecular mechanisms by which acetylcholine and other neurotransmitters inhibit macrophages from responding to ligands of the TLRs and 
other pattern-recognition receptors. Neural circuits are hardwired to occupy a gating position that is critically situated for the modulation of innate immunity, regardless of whether the inciting event is derived from foreign agents produced by microbial invasion or from endogenous molecules that accumulated during ischemic or other sterile injury (Fig. 1). Neurons express the molecular machinery needed to detect foreign and endogenous inflammatory molecules, and new tools can monitor neuronal activity with implantable electrodes and electrode arrays. Such results can be coupled to advanced brain-imaging technologies, including positron emission tomography and magnetic resonance imaging, for the elucidation of the metabolic and functional activity of neural networks. The field of bioinformatics has advanced to the stage at which functional neural data can be combined with classical measures of immune function. Together, this will make it possible to observe signatures of invasion or injury in the nervous system ${ }^{1}$. Combining modalities of neural functioning with immunological end points will map an immunological homunculus, showing how brain regions are organized and function as they receive and transmit information during specific immune responses. New opportunities will arrive for the diagnostic and therapeutic exploitation of neuromodulating devices, perhaps modeled after the deep brain stimulators now being used for the treatment of Parkinson's disease and other disorders.

\section{Innervation of immunity}

The spleen, which has been called "the most important organ for antibacterial and antifungal immune reactivity" 12 , filters aged red blood cells and microorganisms from the blood. Advances in spleen anatomy have elucidated an organization of lymphoid compartments, arteries and nerves in the white pulp, separated from the red pulp by the marginal zone. It is also the main organ source of tumor necrosis factor that accumulates in the blood during endotoxemia. Splenic production of tumor necrosis factor by macrophages in the red pulp and marginal zone is regulated by vagus nerve signals that are transmitted across the celiac ganglion, where the vagus nerve terminates anatomically ${ }^{13}$. Vagus nerve signals are relayed functionally into the spleen via the splenic nerve. In addition to regulating cytokine release by resident macrophages, these neural signals also modulate the trafficking abilities of circulating neutrophils and monocytes, influencing their ability to be recruited to regions of peripheral tissue inflammation ${ }^{14}$ (Fig. 2). How can these phenomena be explained, and what are the implications for understanding the intersection of innate and adaptive immunity and for developing therapeutics?

The answers will probably come from understanding the mechanisms by which information stored or transmitted in the nervous system is transduced to mobile cells of the circulating immune system as they pass through the spleen (Fig. 2). While transiting the spleen, cells of the immune system pass through its vascular matrix in close proximity to nerve endings arising from the splenic nerve. Neurotransmitters released into the splenic interstices downmodulate the inflammatory phenotype of neutrophils, monocytes and lymphocytes so that when exiting the spleen, these cells either fail to be recruited to zones of ischemia or inflammation or fail to mediate damage. This mechanism has implications for understanding disease pathogenesis and the role of neural regulation for inflammatory syndromes initiated or mediated by the trafficking and accumulation of inflammatory cells in peripheral tissues. 
Splenectomy can substantially exacerbate the development of atherosclerosis in apolipoprotein E-deficient mice, yet reconstitution with donor spleen B cells confers protection against the exacerbation of atherosclerosis and diminishes $\mathrm{T}$ cell accumulation in lesions ${ }^{15}$. As neural input to the spleen provides a strong anti-inflammatory signal to passing monocytes and lymphocytes, it will be important to understand how tonic neural signals can modulate the mobilization and recruitment of $\mathrm{T}$ cells to the arterial wall early in the development of atherosclerotic lesions. A similar pathway can be explored for rheumatoid arthritis and other autoimmune diseases, an approach that is conceptually supported by observations showing that the onset of adjuvant arthritis in mice is preceded by depletion of splenic neurons known to mediate the anti-inflammatory activity of vagus nerve- derived signals to the spleen ${ }^{13,16}$. It will be an incremental step from the work now ongoing in this field to understand these mechanisms that unite neuron-derived information with activity of cells of the immune system to regulate the destiny of cells departing the spleen. Unresolving inflammation complicates the effectiveness of anti-inflammatory therapies and is central to the pathogenesis of clinical disease, so the implications for the ability of neural stimulation to enhance the resolution of inflammation are important for developing therapeutics ${ }^{17}$.

\section{Conclusion}

The field of neural control of innate immunity will continue to advance with new information about signal transduction and the role of other neurotransmitter receptors.

Advances in the field of antibody-mediated effects on brain function have identified other critical interactions between the brain and the immune system that influence behavior ${ }^{18}$. Thus, immunologists are now positioned to use tools from neuroscience, physiology and immunology to demonstrate how neural reflexes modulate innate and adaptive immunity. The recognition that the immune system cannot be regarded as autonomous will open new avenues for the pursuit of the discovery of therapies for previously incurable and unpreventable diseases.

\section{References}

1. Tracey KJ. J Clin Invest. 2007; 117:289-296. [PubMed: 17273548]

2. Tracey KJ. Nat Rev Immunol. 2009; 9:418-428. [PubMed: 19461672]

3. Labiner DM, Ahern GL. Acta Neurol Scand. 2007; 115:23-33. [PubMed: 17156262]

4. Watkins LR, Maier SF. J Intern Med. 2005; 257:139-155. [PubMed: 15656873]

5. Watkins LR, et al. Neurosci Lett. 1995; 183:27-31. [PubMed: 7746479]

6. Goehler, Le, et al. Brain Res Bull. 1997; 43:357-364. [PubMed: 9227848]

7. Wang H, et al. Science. 1999; 285:248-251. [PubMed: 10398600]

8. Yang H, et al. Proc Natl Acad Sci USA. 2004; 101:296-301. [PubMed: 14695889]

9. Lotze MT, Tracey KJ. Nat Rev Immunol. 2005; 5:331-342. [PubMed: 15803152]

10. Tsung A, et al. J Exp Med. 2005; 201:1135-1143. [PubMed: 15795240]

11. Yang H, et al. Proc Natl Acad Sci USA. in the press.

12. Mebius RE, Kraal G. Nat Rev Immunol. 2005; 5:606-616. [PubMed: 16056254]

13. Rosas-Ballina M, et al. Proc Natl Acad Sci USA. 2008; 105:11008-11013. [PubMed: 18669662]

14. Saeed RW, et al. J Exp Med. 2005; 201:1113-1123. [PubMed: 15809354]

15. Caligiuri G, Nicoletti A, Poirier B, Hansson GK. J Clin Invest. 2002; 109:745-753. [PubMed: 11901183] 
16. Straub RH, Rauch L, Fassold A, Lowin T, Pongratz G. Arthritis Rheum. 2008; 58:3450-3460. [PubMed: 18975332]

17. Nathan C, Ding A. Cell. 2010; 140:871-882. [PubMed: 20303877]

18. Diamond B, et al. Adv Immunol. 2006; 89:289-320. [PubMed: 16682277] 


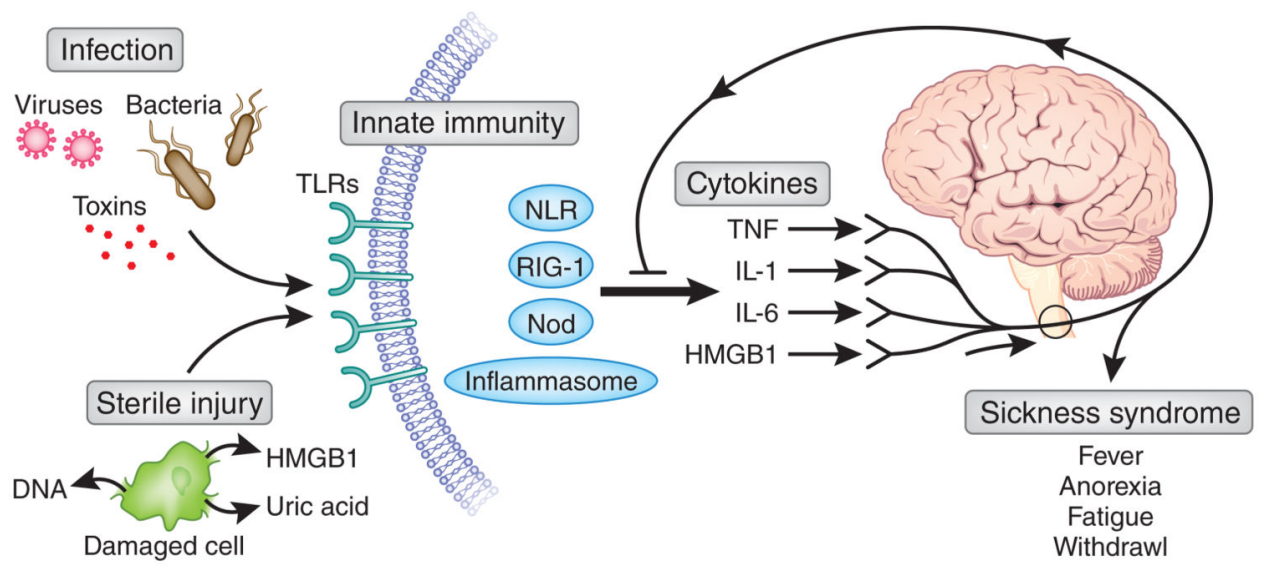

Figure 1.

Foreign products derived from infecting pathogens and endogenous molecules released from ischemic or ruptured cells converge on the innate immune system, which leads to cytokine release. Tumor necrosis factor (TNF), IL-1, IL-6 and HMGB1 interact with neurons to produce a constellation of signs and symptoms known as 'sickness behavior'. Because this is the result of cytokines, it is not possible to distinguish between infectious injury and sterile injury as the activating etiology. The nervous system responds to the presence of cytokines and transmits action potentials via the cholinergic anti-inflammatory pathway to downregulate the progression of inflammation. This is a critical gating or controlling step that leads to the resolution of inflammation and prevents further damage. NLR, Nod-like receptor; RIG, retinoic acid inducible gene; Nod, nucleotide-oligomerization domain protein; HMGB1, high-mobility group protein B1. 


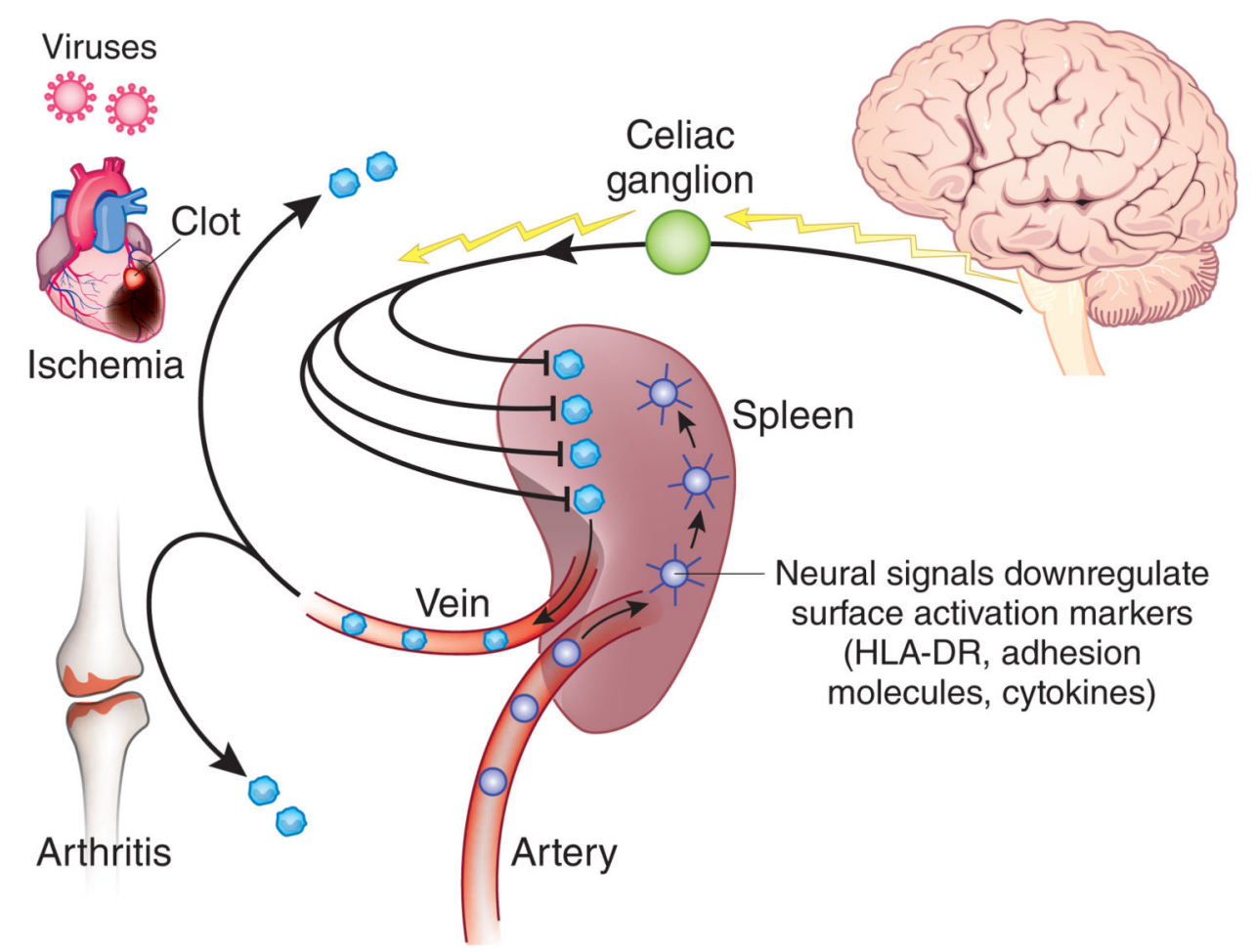

Figure 2.

Neural signals transmitted to spleen down-modulate the inflammatory phenotype of passing lymphocytes and monocytes. The spleen receives $20 \%$ of the cardiac output each minute and is uniquely positioned as a porous vascular bed to connect the information it receives from the brain and to relay this to monocytes, lymphocytes and neutrophils as they pass through its matrix. Neural signals derived from the cholinergic anti-inflammatory pathway downregulate surface expression of major histocompatibility complex HLa-DR molecules and other activation markers, producing an undamaging phenotype to the cells exiting the spleen. Downregulated cells departing the spleen fail to contribute to the progression of damaging inflammation at sites of injury and damage. This mechanism can account for less damage at sites of ischemia or sterile inflammation, as occurs in myocardial ischemia and rheumatoid arthritis. 